\title{
Paul Klee und die Medizin
}

W. Fuchs

\author{
Paul Klee und die Medizin \\ Sonderausstellung vom 31. März bis \\ 9. Oktober 2005 \\ Vernissage am 31. März 2005, 18.15 Uhr \\ Medizinhistorisches Museum der Univer- \\ sität Zürich, Rämistrasse 69, 8006 Zürich \\ Öffnungszeiten: Di bis Fr 13-18 Uhr, \\ Sa und So 11-17 Uhr \\ Eintritt frei
}

Mit der Ausstellung «Paul Klee und die Medizin» greift das Medizinhistorische Museum der Universität Zürich erstmals in einem interdisziplinären Ansatz eine Verbindung von Kunst- und Medizingeschichte bei Paul Klee auf. Dabei wird das Werk von Klee nicht rein ästhetisch, sondern aus seinem historischen Kontext heraus und unter Verwendung von neuesten Forschungsergebnissen präsentiert. Die Zeitspanne der Ausstellung reicht von Klees künstlerischen Anfängen des Jahres 1899 bis zu seinem Tod im Jahre 1940.

Die Ausstellung umfasst folgende vier Themenbereiche:

1. Von der Menschenanatomie zur Bildanatomie;

2. Pathologisierung von Klees Kunst;

3. Klees Ärztebeziehungen;

4. Klees letzte Krankheit.

\section{Von der Menschenanatomie zur Bildanatomie}

Dieser Teil der Ausstellung befasst sich zunächst mit der künstlerischen Ausbildung von Paul Klee in Akt- und Anatomiezeichnung in München und Bern. Beispielhaft für Klees Aktzeichnungen zeigen wir die Arbeit Ein berüchtigtes Mannweib von 1899. Nach seiner Italienreise besuchte Klee im Wintersemester 1902/03 am Anatomischen Institut der Universität Bern Vorlesungen über «Plastische Anatomie» bei Professor Hans Strasser.

Der Ausstellungsbesucher kann Klees anatomische Beobachtungs- und Darstellungsfähigkeit anhand von zwei Anatomiezeichnungen selbst überprüfen. Ergebnis dieses Anatomiestudiums bildete 1903 die Radierung Jungfrau (träumend), auch als Jungfrau im Baum bekannt, die sich als «Aktkarikatur» vom vorherrschenden Akademiegeschmack abgrenzte.

Der von Klee beschriebene Übergang von der «Menschenanatomie» zur «Bildanatomie» zeigt sich besonders bei den beiden Aquarellen Anatomie der Aphrodite und Flügelstücke zu 1914.45. In einer digitalen Animation wird sowohl der medizinhistorische wie auch der kunsthistorische Hintergrund von Klees Arbeitsprozess des Übermalens, Zerschneidens und Neukombinierens vorgestellt.

Die späte Zeichnung Neu gerichtet - ein Jahr vor dem Tod des Künstlers entstanden - kann im Rahmen von Klees Darstellungen fragmentierter Körper im Zusammenhang mit Jacques Lacans Theorie über das Spiegelstadium (1936) verstanden werden. Das Werk bietet aber auch Einblick in Klees Auseinandersetzung mit dem zeitgenössischen Surrealismus.

\section{Pathologisierung von Klees Kunst}

Von Paul Klees kulturkritischem Ideal der «Geisteskrankenkunst» und der diffamierenden Gleichsetzung von Autor und Werk zeugt eine weitere Abteilung der Ausstellung. Hier wird gezeigt, wie Klee zunächst das kreative Schaffen von Geisteskranken zu einem seiner künstlerischen Ideale erhob und wie er in der Folge - wie viele andere Künstler der Moderne - ins Blickfeld der psychiatrischen Wissenschaft geriet und zunehmend psychopathologisiert wurde. Kritiker und Ärzte zogen Rückschlüsse von Klees Kunst auf dessen Geistes- und Gesundheitszustand.

In diesem Teil erfährt der Museumsbesucher, wie zur Zeit des Nationalsozialismus die Pathologisierung und die damit einhergehende Diffamierung Klees und vieler seiner Kollegen als «entartete Künstler» in der Wanderausstellung «Entartete Kunst» den absoluten Höhepunkt erreicht (Die Heilige vom innern Licht). In seinem Schweizer Exil erlebte Klee subtilere Formen der Ausgrenzung, etwa in der Kritik der «Neuen Zürcher Zeitung». Auf die Pathologisierung von Klees Spätwerk wird ganz zum Schluss des Ausstellungsparcours in der Abteilung «Klees letzte Krankheit» eingegangen. 


\section{Abbildung 1}

«Der [böse] Geist der Medizin ist leicht zu fassen...» (Paul Klee nach Goethe, 1901). Paul Klee: Ex Libris L. Michaud, 1901. Radierung auf Papier, $15,8 \times 11,6 \mathrm{~cm}$. Zentrum Paul Klee, Bern. Leihgabe aus Privatbesitz.

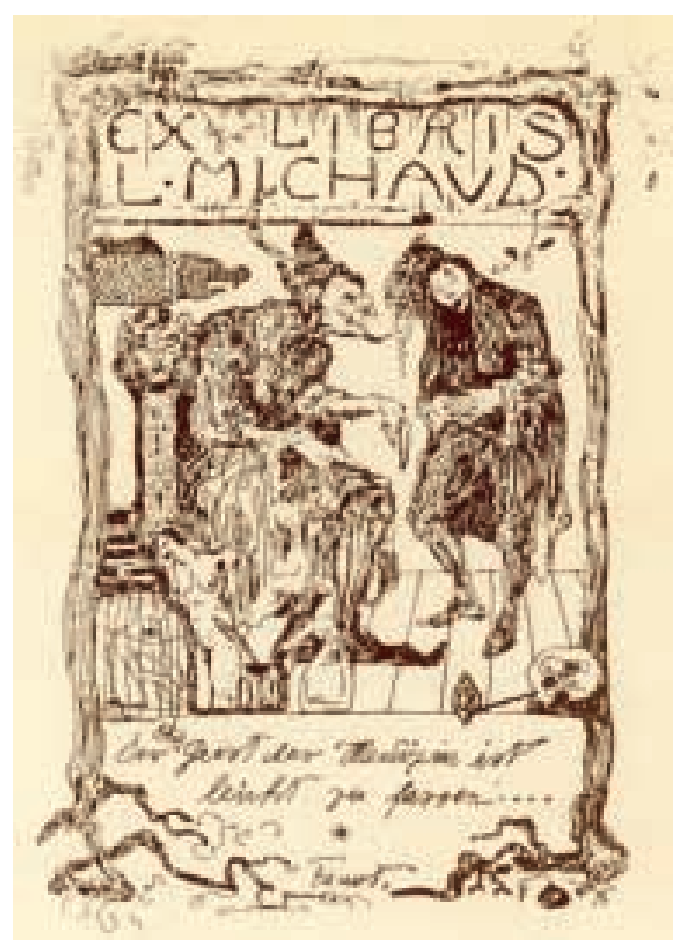

Als Intermezzo zu diesem Thema ist eine Vitrine mit zwei Apparaten gedacht: Die Zeichnung Psychoregistrierapparat von Paul Klee wird Carl Gustav Jungs «Lügendetektor» zur Messung von «psycho-galvanischen Phänomenen» gegenübergestellt. In demselben Schaukasten präsentieren wir Rainer Maria Rilkes «Ur-Geräuschapparat» und erklären, was dieser mit Klee zu tun hat.

\section{Klees Ärztebeziehungen}

Hier wird der Schwerpunkt nicht ausschliesslich auf die Ärzte gelegt, die Klee zuletzt behandelt haben, sondern es sind Mediziner aus allen Lebensabschnitten vertreten - so Klees bester Freund, der Neurologe Fritz Lotmar, der Schul- kamerad und spätere Lausanner Internist und Ordinarius Louis Michaud (Ex Libris L. Michaud, 1901), der Dermatologe Felix Lewandowsky, mit dem Klee musiziert hat, sowie sein Schwiegervater, Impfarzt Dr. med. Ludwig Stumpf, mit dem ihn ein gespanntes Verhältnis verband.

Die Ärzte, die Klee während seiner letzten Krankheit von 1935 bis 1940 behandelt haben Gerhard Schorer (Hausarzt), Oskar Nägeli (Spezialist), Hermann Bodmer (Kurarzt) und Theodor Hämmerli (Herzspezialist) - sind in der Abteilung «Klees letzte Krankheit» zu finden, der Berner Anatomielehrer Hans Strasser eingangs der Abteilung «Von der Menschenanatomie zur Bildanatomie».

\section{Klees letzte Krankheit}

Das eigentliche Herzstück der Ausstellung bildet die massstabgetreue begehbare Rekonstruktion von Paul Klees Berner Atelier unter Verwendung von originalen Möbeln, Architekturteilen und Photographien. Erst durch die physische Erfahrbarkeit des Ateliers wird dem Besucher bewusst, in welch bescheidenen räumlichen Verhältnissen Klee in seinem Berner Exil gewirkt hat. Sein Spätwerk ist vertreten durch die Originalzeichnung Ein Kranker macht Pläne und eine Kopie des Tafelbildes Vorhaben.

Klees letzte Krankheit wird aus der Sicht von Patient, Familie und Freunden in einer vertonten Bildvorführung von den beiden Schauspielern Barbara Ahren und Frank Demenga nacherzählt. Diese Patientengeschichte findet eine Erweiterung mit einem Porträt seines Vertrauensarztes Gerhard Schorer, mit eindrücklichen Wachsmodellen von Klees posthum diagnostiziertem Krankheitsbild Sklerodermie und den historischen Quellen zu Klees Leiden, an dem er 1940 verstarb.

Die Aufarbeitung der letzten Krankheit von Paul Klee in Berücksichtigung seines personellen und künstlerischen Umfeldes und der Medizingeschichte könnte eine Basis für weiterführende Interpretationen des im Berner Exil entstandenen Spätwerks darstellen. 